\title{
Über die Wirkung von $\iota$-Bestrahlung auf die Anklingung eines ZnSCu-Phosphors
}

\author{
Von Fritz Bandow * \\ (Z. Naturforschg. 3a, 16-20 [1948]; eingegangen am 26. September 1947)
}

\begin{abstract}
Messungen der Anklingung des Nachleuchtens führen zur Festlegung einer Anklingungsgröße $A$. Dadurch ergibt sich ein quantitatives Maß für den Einfluß von $\alpha$-Bestrahlung. $A$ hängt bei dem mit $\alpha$-Strahlen behandelten $\mathrm{ZnSCu-Phosphor} \mathrm{„Grün} \mathrm{N“} \mathrm{vom}$ Erregungszustand der langdauernden Zentren ab. Wenn diese unerregt sind, ist die Anklingung der kurzdauernden Zentren stark verzögert.
\end{abstract}

1. ZnS-Phosphore werden durch die Einwirkung von $\alpha$-Strahlen stark verändert, und zwar in charakteristischer, von der Wirkung des Drukkes verschiedener Art. Die Verminderung (,Zerstörung") der Lumineszenzfähigkeit ist schon lange bekannt (E. Marsden, E. Rutherf o r d). Neu entdeckt wurde in den letzten Jahren die erhöhte Tilgbarkeit des Restleuchtens durch Bestrahlung mit Rot und Ultrarot und eine Verzögerung der Anklingung des Restleuchtens, während die Abklingung beschleunigt ist ${ }^{\mathbf{1 , 2}}$. Die gegenläufige Wirkung auf das Anklingen und das Abklingen ist auffällig, denn in natürlicher Weise gehören schnellere Anklingung und schnellere Abklingung zusammen. Am leichtesten ist dies bei den radioaktiven Prozessen zu übersehen, bei denen Verwicklungen durch äußere Einflüsse fehlen. Bei den Phosphoren ist in der Hauptsache der gleiche Zusammenhang beobachtet worden ${ }^{3}$. Abweichungen von dem normalen Verhalten zeigen an, daß $Z$ wischenmechanismen wirksam werden oder daß sich die Präparate während der Beobachtung verändern, z. B. in ihren Absorptionseigenschaften. Die Aufklärung dieser Verhältnisse ist in gleicher Weise wichtig für das Verständnis des Phosphoreszenz- und des Zerstörungsvorgangs. Im Rahmen der umfassenden Untersuchungen, die Hr. Prof. A. B e c k e r mit

* Mannheim, Max-Joseph-Str. 7.

1 Zahlreiche Arbeiten, besonders in den Ann. Physik von Bd. 34 [1939] an. Ferner die vielseitige Untersuchung von Fritz B e cker, Z. Naturforschg. 2a, 100 [1947]. Hier sind auch Ergebnisse von mir angeführt. Ich bemerke dazu folgendes: $\mathrm{Zu}$ S. 103, Anm. 5: Die Messungen, auf denen diese Angabe beruht, sind nicht durch Streulicht gestört; es handelt sich um eine starke Wirkung. Zu S. 107, rechts oben: Hier liegt ein Mißverständnis vor; quantitative Messungen habe ich nur bei 800 bis 2000 Volt angestellt, bei 30000 Volt nur qualitative Beobachtungen. seinen Mitarbeitern im physikalischen Institut der Universität Heidelberg durchgeführt hat, ${ }^{1,2}$ habe ich es Anfang 1945 unternommen, die Anklingungsverzögerung für das Nachleuchten quantitativ zu ermitteln ${ }^{4}$, und zwar an dem sehr bekannten ZnSCu-Phosphor „Grün N“ (mit geringem Zusatz von $\mathrm{ZnO}$ und CdS hergestellt), der schon $\mathrm{zu}$ vielen grundlegenden Messungen wissenschaftlicher und technischer Art benutzt worden ist. Die quantitative Erfassung der neuen Erscheinungen ist die Vorbedingung für weitere vergleichende Untersuchungen (Abhängigkeit von der Zusammensetzung der. Präparate, von der Stärke der $\alpha$-Einwirkung usw.) und damit die Voraussetzung für die Deutung. Das Ziel ist selbstverständlich die Einordnung der experimentellen Befunde in größere Zusammenhänge, die aus dem Erscheinungsbild abzuleiten sind.

\section{Experimentelles}

2. Ich benutzte 4 Phosphorproben: 1 . Grün N, unbehandelt; 2. Grün N, durch Zerreiben auf 1/5 druckzerstört; 3. Grün N, mit $2100 \mathrm{mg}$ · Stde. Ra.äq./g bestrahlt; 4. zum vorläufigen Vergleich einen CaSBi-Phosphor mit geringem Schwermetallgehalt (weit ,unternormal"; eigene Präparation, nach Len ard und Klatt). - Erregung durch eine Quecksilberlampe HgQ 300 mit Dunkelkolben; Abstand $28 \mathrm{~cm}$; senkrechter Strahleneinfall. Fernhalten langwelliger tilgender Strah-

2 Verzögertes Anklingen: A. B e c k e r, I. S c h a p e r, Ann. Physik 42, 498 [1943].

${ }^{3}$ P. Lenard, F. Schmidt u. R. Tom a s c h e k, Handb. Exper.-Physik XXIII, S. 41 und 766, 1928. Die heutigen Anschauungen über den Anklingungsvorgang sind gegenüber Lenards Vorstellungen teilweise verändert, die allgemeine Grundbeziehung bleibt aber erhalten.

${ }^{4}$ Eine Zusammenstellung meiner Versuche an Phosphoren in der Zeit von Ende 1943 bis Anfang 1945 habe ich im Herbst 1945 bei der Heidelberger Akademie der Wissenschaften hinterlegt. 
len durch eine 2-proz. $\mathrm{CuSO}_{4}$-Lösung, $5 \mathrm{~cm}$ Schichtdicke. Das ist der ,Normalwert“ der erregenden Intensität. Durch Drahtnetze konnte eine Abschwächung bis auf 1/50 des ,Normalwertes“ vorgenommen werden. Tilgung durch eine Wendellampe Osram Nr. 8031 für 8 Amp., 12 Volt Belastung, betrieben mit 7 Amp.; Abstand $23 \mathrm{~cm}$; Filter UG 7 von Schott, Jena, $2 \mathrm{~mm}$ dick, senkrechter Strahleneinfall. - Der Phosphor befand sich in einem kleinen, mit dünnem Deckglas bedeckten, heizbaren Metalltrog in ,großer" Schichtdicke. Der Trog war auf einer optischen Bank mit entsprechenden Anschlägen vor der Erregungs- und Tilgungslampe und Zelle verschiebbar. - Die Lichtsumme wurde für ausgewählte Bereiche der Abklingzeit mit lichtelektrischer Alkalizelle und Elektrometer gemessen. Ausgeheizt wurde nur zur Vorabklingung (vgl. Abschn. 4 u.5) und für die Messungen zu Tab.1, nicht aber für die Messungen der Größe $A$ in der Haupttabelle 2.

3. Ein Maß für die Anklingung liefert der Ausdruck $A=\frac{L_{t}}{L_{\infty}} \cdot \frac{1}{t}$. Dabei bedeutet $L_{t}$ die Lichtsumme in dem untersuchten Zeitbereich nach $t$ sec Erregungsdauer, $L_{\infty}$ die Lichtsumme im gleichen Zeitbereich nach so langer Erregung, daß eine weitere Steigerung praktisch nichts mehr ausmacht. $L_{t}$ und $L_{\infty}$ gehören jeweils zur gleichen erregenden Intensität. Bei der erregenden Intensität ${ }^{1 / 50}$ lag $L_{\infty}$ um mehr als die Hälfte unter der vollen Lichtsumme, welche der Phosphor für den gewählten Bereich der Abklingung aufspeichern konnte. Wenn das Verhältnis $L_{t} / L_{\infty}=1 / 2$ gewählt wird, bedeutet $t$ eine „Halbwertszeit der Anklingung" und die Anklinggröße $A$ wird $=\frac{1}{2 t}$. Die genauere Form der Anklingkurve bleibt offen.

4. Versuchsgang: Für verschiedene erregende Intensitäten, angegeben in Bruchteilen der „Normalintensität", wurde $L_{\infty}$ für bestimmte Bereiche der Abklingkurve ermittelt. Ebenso wurde $L_{t}$ gemessen für verschiedene Erregungszeiten $t$, die so gewählt waren, daß das Verhältnis $L_{t} / L_{\infty}$ ungefähr $=1 / 2$ war. Daraus wurde $A$ berechnet. Der Erregungszustand der Phosphore wurde für die Messungen von $L_{t}$ dadurch variiert, daß zwischen eine Erregung von ,unendlich" langer Dauer und die zur eigentlichen Messung gehörige Teilerregung von gemessener, beschränkter Dauer eine Vorabklingung eingeschaltet wurde. Dafür gibt es drei Möglichkeiten: 1. Abwarten bei Zimmertemperatur. 2. Ausheizen. Dies wurde, wenn es überhaupt angewandt wurde, so bemessen, daß der Phosphor in den völlig unerregten Zustand

\begin{tabular}{|c|c|r|r|r|r|}
\hline \multirow{2}{*}{ Phosphor } & \multicolumn{2}{|c|}{ Wartezeit } & \multicolumn{3}{|c|}{ Tilgungszeit } \\
\cline { 2 - 3 } \cline { 5 - 6 } & $1 \mathrm{~h}$ & $21 \mathrm{~h}$ & $1 \mathrm{~min}$ & $4 \mathrm{~min}$ & $12 \mathrm{~min}$ \\
\hline Grün N, un- & & & & & \\
behandelt. & 73 & $\sim 20$ & 81 & 24 & $\sim 6$ \\
CaSBi ... & 900 & 700 & 600 & 260 & 80 \\
\hline
\end{tabular}

Tab. 1. Relative Restlichtsumme, durch Ausheizen gemessen.

Die Lichtsumme zwischen 30 und 90 sec nach Schluß der Erregung = 100 gesetzt. Erregung mit 1/50 Intensität.

überging. Bei der $\boldsymbol{\alpha}$-bestrahlten Probe habe ich aber von dieser Behandlung absehen müssen, um nicht das wertvolle, nur in beschränkter Menge verfügbare Präparat bleibenden Veränderungen auszusetzen. 3. Tilgen (bzw. Auslöschen). Zur Beurteilung der bei den Hauptergebnissen in Tab. 2 eingetragenen Wartezeiten und Tilgungszeiten habe ich in Tab. 1 einige relative, durch Ausheizen gemessene Restlichtsummen zusammengestellt. Dabei ist die zwischen 30 und $90 \mathrm{sec}$ nach Schluß der Erregung (mit 1/50 Intensität) ohne Ausheizen abklingende Lichtsumme $=100$ gesetzt. Druckzerstörung ergibt nach bisheriger Kenntnis keine Veränderung der Abklingkurve (H. K u p pen heim u.a.), $\alpha$-Bestrahlung ergibt eine Beschleunigung der Abklingung (E. Streck u. a.), wenigstens in dem quantitativ verfolgbaren und quantitativ überwiegenden Anfangsbereich. Nach $21 \mathrm{~h}$ Abwarten ist Grün N schon weitgehend abgeklungen. Der benutzte CaSBi-Phosphor zeigte dagegen ein sehr viel langsamer abklingendes und im ganzen auch weniger stark tilgbares Nachleuchten (die langdauernden Zentren sind weniger stark tilgbar als die kurzdauernden: P. Len ard, F. B a nd ow).

\section{Ergebnis se}

5. In Tab. 2 sind außer den $A$-Werten einige Werte für eine Auslöschungs(Tilgungs)-größe $\sigma$ eingetragen, die bei diesen Versuchen nebenbei erhalten wurden. $\sigma$ mißt die Tilgbarkeit des Nachleuchtens für den untersuchten Bereich (30 bis 90 sec nach Schluß der Erregung); $\sigma$ ist der zur Halbierung der Lichtsumme notwendigen Tilgungszeit umgekehrt proportional ${ }^{5}$. Die Zahlen stimmen befriedigend mit früheren, photographisch ermittelten Werten überein ${ }^{6}$. Vor allem ist wieder die Verstärkung der langwelligen

${ }^{5}$ F. B a n d ow, Ann. Physik 6, 445 [1930] (nach dem Vorgang von P. Le n a r d u. H. M o s e r).

${ }^{6}$ F. B a n d ow, Ann. Physik 1, 399 [1947]. 


\begin{tabular}{|c|c|c|c|c|c|c|c|c|c|}
\hline \multicolumn{3}{|c|}{ Präparat } & \multicolumn{2}{|c|}{$\begin{array}{l}\text { Grün N un- } \\
\text { behandelt }\end{array}$} & \multicolumn{2}{|c|}{$\begin{array}{c}\text { Grün N auf } \\
1 / 5 \text { druck- } \\
\text { zerstört }\end{array}$} & $\begin{array}{c}\text { GrünN } \\
a \text {-zer- } \\
\text { stört }\end{array}$ & \multicolumn{2}{|c|}{$\mathrm{CaSBi}$} \\
\hline \multicolumn{3}{|c|}{$\begin{array}{l}\text { Tilgungs- } \\
\text { größe } \sigma\end{array}$} & \multicolumn{2}{|c|}{0,03} & \multicolumn{2}{|c|}{0,06} & 1,0 & \multicolumn{2}{|c|}{0,06} \\
\hline \multicolumn{3}{|c|}{$\begin{array}{l}\text { Hg-Inten- } \\
\text { sität (grobe } \\
\text { Werte) }\end{array}$} & $1 / 12$ & $1 / 50$ & $1 / 12$ & $1 / 50$ & $1 / 12$ & \multicolumn{2}{|c|}{$1 / 12 \quad 1 / 25$} \\
\hline 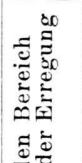 & | & \begin{tabular}{|c}
$\mathrm{h}$ \\
0,5 \\
1 \\
3 \\
21
\end{tabular} & 0,11 & 0,039 & 0,10 & 0,032 & $\begin{array}{l}0,11 \\
0,09 \\
0,05 \\
0,04\end{array}$ & 0,028 & 0,013 \\
\hline$\Xi \stackrel{\emptyset}{\Xi}$ & & $\begin{array}{l}\text { Aus- } \\
\text { izen }\end{array}$ & 0,10 & 0,034 & & 0,022 & & 0,011 & 0,0065 \\
\hline 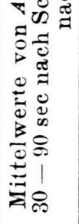 & 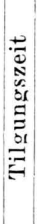 & $\begin{array}{c}\text { sec } \\
1 \\
2 \\
5 \\
60 \\
240 \\
720\end{array}$ & 0,105 & 0,034 & $\begin{array}{l}0,08 \\
0,08\end{array}$ & $\begin{array}{l}0,025 \\
0,022\end{array}$ & $\begin{array}{l}0,09 \\
0,05 \\
0,03 \\
0,021 \\
0,018 \\
0,017\end{array}$ & $\begin{array}{l}0,03 \\
0,02\end{array}$ & $\begin{array}{l}0,016 \\
0,0105 \\
0,008 \\
0,0065\end{array}$ \\
\hline
\end{tabular}

Tab. 2. Ergebnisse der Anklingungsmessungen.

Tilgung durch die Einwirkung der $\boldsymbol{\alpha}$-Strahlen sehr deutlich. (Für einen Rest langdauernder Zentren ist $\sigma$ noch unbekannt.)

6. Besprechung der Anklingungswerte: Durch ausgiebige Vorabklingung wird $A$ bei unbehandeltem Grün N kaum beeinflußt - das ist zugleich eine ganz unmittelbare Probe darauf, daß die Wirkung bei dem mit $\alpha$-Strahlen behandelten Präparat nicht durch Fehlerquellen entstellt ist! Die geringen Unterschiede der Werte für unbehandeltes Grün N liegen an der Grenze der erzielten Meßgenauigkeit $( \pm 5 \%)$. Dagegen ist die Wirkung bei der mit $\alpha$-Strahlen behandelten Probe, deren Lichtsumme im Meßbereich auf 1/12 vermindert ist, außerordentlich stark. Während der Anfangswert von $A$ nicht verändert ist, tritt eine Senkung um den Faktor 6 bei Neuerregung nach langer Ultrarotbestrahlung oder nach langer $\mathrm{Ab}$ klingzeit ein. Das ist ein zahlenmäßiger Ausdruck jür die Verzögerung der Anklingung des Nachleuchtens, die durch die $\alpha$-Strahlen neu hervorgerufen wird. - Messungen für den Bereich 5 bis

\footnotetext{
; Statt von Zentren verschiedener Energieisolation oder verschiedener Dauer (P. L e n a r d) wird in den neueren Phosphoreszenztheorien (N. R i ehl, M. Schön, W. Schot tky u. a.) von Haftstellen oder Anlagerungsstellen oder -termen mit verschiedener Energie oder Bindungsfestigkeit der aufgespeicherten Elektronen gesprochen; vgl. die Zusammenstell ung von K. B i r u s, Ergebn. exakt. Naturwiss. 20, $183[1942]$
}

15 sec nach Schluß der Erregung lieferten ein gleichartiges Ergebnis. -

7. Meine Messungen liefern also den gesuchten Zahlenwert; sie zeigen außerdem, daß hier eine neue Erscheinung vorliegt, die eingehenderer Betrachtung bedarf: Auf die Anklinggeschwindigkeit (des Mitleuchtens und) des hier quantitativ untersuchten Nachleuchtens hat der Erregungszustand von sehr langdauernden Zentren ${ }^{7}$ Ein$f u \beta$, die selbst nicht einen merklichen Beitrag zum Nachleuchten dieses Meßbereichs liefern. Zur Begründung dieser Angabe dienen außer den Zahlen der Tab. 1 noch folgende Prüfungen: für das $\alpha$-bestrahlte Präparat war die in 1 min ohne Ausheizen freiwerdende Lichtsumme nach $1 \mathrm{sec}$ Tilgung bei den angewandten Versuchsbedingungen auf weniger als $1 / 5$, nach $1 \mathrm{~h}$ Abwarten auf weniger als ${ }^{\mathbf{1}} / \mathbf{1 0}$ gesunken, bei dem unbehandelten Grün N nach $1 \mathrm{~h}$ Abwarten auf weniger als 1/50. Und doch ist, wie die Werte der Tab. 2 zeigen, die $A b$ nahme von $A$ bei dem $\alpha$-bestrahlten Grün $\mathrm{N}$ in der Hauptsache an größere Tilgungs- oder Wartezeiten geknüpft. Obgleich die Anzahl der langdauernden Zentren geringer ist als die Anzahl der im Meßbereich abklingenden Zentren, bestimmt doch der Erregungszustand der langdauernden Zentren die Geschwindigkeit der Anklingung der kurzdauernden Zentren. Wenn die langdauernden erregt sind, klingen die kurzdauernden schneller an. Die Erregung des $\alpha$-bestrahlten Phosphors hat bei Beginn vom ganz unerregten Zustand aus zunächst eine geringere Quantenausbeute. Von einer größeren Trägheit des Anklingvorgangs im ganzen kann nicht gesprochen werden, denn die $A$-Werte liegen nach kurzer Warte- oder Tilgungszeit nicht unter den normalen.

8. Die druckzerstörte Probe weicht wenig von dem Verhalten des unbehandelten Phosphors ab. Die schon in vielen Versuchen nachgewiesene Verschiedenheit der beiden Zerstörungsvorgänge ${ }^{8}$ kommt, wenn nicht im Grundsätzlichen, so doch im quantitativen Ausmaß der Veränderung auch hier wieder zum Ausdruck.

9. Bei dem CaSBi-Phosphor ist die Anklingung viel langsamer als bei Grün N (kleineres $A$ ). Das paßt mit der langsameren Abklingung, die u. a. durch die Zahlen der Tab. 1 belegt wird, zusammen im Sinne des anfangs erwähnten Grundgesetzes. Die Abhängig-

$8 \mathrm{Vgl}$. besonders A. B e c k e r u. I. S chaper. Ann. Physik 42, 297 u. 487 [1943] und A. B e cker u. F. B e cker, Ann. Physik 43, 598 [1943]. 
keit der Anklingung vom Erregungszustand ist groß. Die Frage, wie weit ein allgemeinerer, grundsätzlich wichtiger Einfluß des Grundmaterials und der hier verhältnismäßig großen Inhomogenität der Zentrenverteilung vorliegt, ist bis zur Durchführung weiterer Versuche zurückzustellen; dabei ist natürlich auch das Verhalten des Mitleuchtens und des durch Elektronen erregten Leuchtens mit zu berücksichtigen ${ }^{9}$.

10. Gibt es eine besondere Nachwirkung von ultraroter Bestrahlung (,Tilgungsnachwirkung") ? Die Anklingverzögerung (Verkleinerung von $A$ ) bei dem $\alpha$ zerstörten Phosphor Grün N tritt, wie bereits hervorgehoben, nach Übergang in den unerregten $\mathrm{Zu}$ stand in Erscheinung. Ein Zeichen dafür, daß die Ultrarotbestrahlung einen besonderen, mit Trägheit abklingenden Zustand des Phosphors hervorruft, ist nicht erkennbar. Erhitzen solcher Präparate nach der tilgenden Bestrahlung hat keinen Einfluß auf die Erscheinungen. Auch tritt (nach Beobachtungen von A. B e c k e r) kein Unterschied hervor, wenn ein Teilfeld des Phosphors im völlig unerregten Zustand mit langwelligem Licht bestrahlt wird. Wenn ein Teilfeld eines nicht ganz unerregten Präparates mit Ultrarot bestrahlt wird, erscheint es bei Neuerregung zunächst dunkler als seine Umgebung, denn in diesem Teil wird der Erregungszustand durch die Tilgung herabgesetzt und $A$ verkleinert. Dieser Unterschied wird durch Ausheizen zum Verschwinden gebracht (A. B e cker), weil dadurch das ganze Präparat in den unerregten Zustand übergeht. Meine Messungen stehen mit den angeführten Beobachtungen in Einklang.

11. Daf die Anklingung des Nachleuchtens (d-Prozeß) und auch des Mitleuchtens bei Beteiligung des d-Prozesses nicht momentan erfolgen kann, ist energetisch selbstverständlich. Entsprechendes gilt für die Tilgung; ihre Trägheit kommt bei meinen Messungen darin zum Ausdruck, daß trotz der Erhöhung der Tilgungsgröße eine erhebliche Tilgungsdauer notwendig ist, um die volle Anklingverzögerung hervorzurufen. Jedoch ist die Trägheit der Tilgung für den $\alpha$-zerstörten Phosphor geringer als für den unbehandelten, weil durch die $\alpha$-Strahlen eine Verstärkung der Tilgung im ganzen und außerdem eine Beschleunigung der Abklingung hervorgerufen wird ${ }^{\mathbf{1 0}}$.

\section{Besprechung}

12. Eine modellmäßige Deutung aller Ergebnisse und die Einordnung in eine Gesamtvorstellung erfordert eine planmäßige Verbreiterung der experimentellen Grundlage. Ich will aber schon jetzt darauf hinweisen, daß eine Vergleichsmöglichkeit mit der Proportionalitätsabweichung zwischen erregender Intensität und Intensität des

9 Vgl. u. a. die Methode ron A.Schle ed e u. B. B a rtels, Z. techn. Physik 19, 364 [1938]. Sie fanden später wesentliche Unterschiede im Verhalten von CaS und $\mathrm{ZnS}$.
Mitleuchtens besteht. Diese wurde von J. H. Gisolf und F. A. Kroeger ${ }^{11}$ und Rieh $1^{12}$ entdeckt (vgl. auch ${ }^{13}$ ). An ZnSCdS-Ag-Phosphoren bei Zimmertemperatur und an $\mathrm{Cu}-\mathrm{Phosphoren}$ bei erhöhter Temperatur ${ }^{12}$ steigt die Intensität des Mitleuchtens steiler an als die erregende Intensität; die Quantenausbeute der Erregung ist bei geringen Intensitäten, welche im Gleichgewichtszustand nur eine schwache Erregung des Phosphors bewirken, kleiner als 1. Analog zeigten meine oben erläuterten Versuche, daß die Quantenausbeute der Erregung des Nachleuchtens bei Beginn vom ganz unerregten Zustand aus zunächst gering ist; ein erheblicher Teil der Absorptionsakte führt nicht zur Emission - weder im Mitleuchten noch in dem zunächst aufgespeicherten und erst später allmählich abklingenden Nachleuchten die Energie wird im Phosphor zerstreut. Eine erste Vergleichsmöglichkeit ist also gegeben.

13. In einer von mir untersuchten Reihe von ZnSCdS-Ag-Phosphoren war die Proportionalitätsabweichung nur bei 20 und 30\% CdS stark ausgeprägt. Diese beiden Phosphore waren außerdem die einzigen der Reihe mit kräftiger langwelliger Tilgung des Mitleuchtens. Die Wahl der erregenden Frequenz hatte starken Einfluß auf die Erscheinungen: die langwellige Tilgung und die Proportionalitätsabweichung waren bei Erregung mit $\mathrm{Hg} 435 \mathrm{~m} \mu$ größer als bei Erregung mit $\mathrm{Hg} 405 \mathrm{~m} \mu^{14}$. Diese Erregungsfrequenzen fallen bei dem angegebenen Cd-Gehalt in den Grenzbereich der Grundgitterabsorption (vgl. Birus s${ }^{7}$ ). Die gemeinsame Ursache der besonderen Erscheinungen könnte eine Inhomogenität der Struktur und der Lumineszenzprozesse sein. Ich vermute, daß die $\alpha$-Strahleinwirkung, welche eine Tilgungssteigerung und die Veränderung der Anklingverhältnisse hervorruft, eine Inhomogenität im Aufbau der Phosphore verursacht, die verschiedene, sich beeinflussende Lumineszenzvorgänge bedingt. Dabei sind die Lumineszenzerscheinungen

10 Der elektrische Tilgungseffekt kann daher beim $\alpha$-zerstörten Phosphor im Vergleich zum unzerstörten ,fast momentan“ erscheinen: F. Goos, Ann. Physik 37, 85 [1940]; im übrigen hängen die elektrischen Eigenschaften der Phosphore zum Teil nicht unmittelbar mit den Leuchteigenschaften zusammen: F. Goos, ebenda und Ann. Physik 39, 281 [1941]; dort weitere Zitate.

11 J. H. Gisolf u. F. A. K r o e ger, Physica 6, 1101 [1939].

12 N. R i e h l, Z. techn. Physik 20, 152 [1939].

13 Handb. Exper.-Physik XXIII, S. 706, 707, 723.

14 Vgl. F. B e cke r ${ }^{1}$. 
- wie es allgemeiner Erfahrung entspricht viel empfindlichere Nachweismittel als Strukturuntersuchungen mit Röntgeninterferenzen ${ }^{15}$.

14. Meine photometrischen Messungen des Mitleuchtens mit Sperrschichtphotoelement und Spiegelgalvanometer zeigten, daß an Grün $\mathrm{N}$ bei Zimmertem-

${ }_{15}$ Durchgeführt von F. We cke r, Ann. Physik 42, 561 [1943]. Vgl. dazu in allgemeiner Hinsicht P. W. B r idgman, Physic. Rev. 57, 237 [1940]. peratur keine Proportionalitätsabweichung auftritt, wenn die erregende Intensität in der Nähe der in Abschn. 2 angegebenen „Normalintensität“ liegt. Die entsprechenden Versuche an der $\alpha$-zerstörten Probe führten noch nicht zu einem sicheren Ergebnis. Ich vermute, daß bei kleinsten Intensitäten eine Proportionalitätsabweichung als Folge der $\alpha$-Bestrahlung neu auftritt, oder daß sie (im Sinne der Vorstellungen

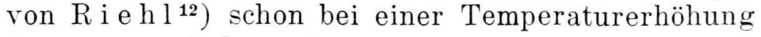
deutlich wird, die nicht so groß zu sein braucht wie bei dem unbehandelten Phosphor.

\title{
Leitfähigkeit und Hall-Effekt von Germanium
}

\author{
Von Walter Ringer und Heinrich Welker \\ Aus dem Physikalisch-chemischen Institut der Universität München \\ (Z. Naturforschg. 3a, 20-29 [1948]; eingegangen am 25. Juli 1947)
}

\begin{abstract}
Im experimentellen Teil der Arbeit wurden an unlegierten und mit Kupfer legierten Germaniumpräparaten, welche im Hochvakuum gegossen worden waren, Leitfähigkeit und Hall-Effekt-Messungen durchgeführt. Dabei wurde an dem reinsten Germaniumpräparat mit einem Verunreinigungsgehalt von ungefähr $10^{-3}$ Gew.- $\%$ der spezif. Widerstand $\varrho=0,86 \Omega \mathrm{cm}$ und die Hall-Konstante $R=2000 \mathrm{~cm}^{3} / \mathrm{Amp}$. sec gefunden, woraus sich eine Elektronenkonzentration von $n=3 \cdot 10^{15} \mathrm{~cm}^{-3}$ und eine Elektronenbeweglichkeit $b=2000 \mathrm{~cm} \mathrm{sec}^{-1} / \mathrm{Volt}_{\mathrm{cm}-1}$ errechnet. Für die Präparate mit einem Fremdstoffgehalt $<0,1$ Gew.- $\%$ ist die Elektronenbeweglichkeit konstant und nimmt obigen Wert an, so daß die Leitfähigkeit in diesem Bereich nur noch von der Elektronenkonzentration abhängig ist, welche ihrerseits wieder als dem Fremdstoffgehalt proportional erscheint. Für reinstes Germanium ist demnach ein um Größenordnungen höherer Widerstand als der hier gemessene zu erwarten.

Im theoretischen Teil der Arbeit wird gezeigt, wie die aus den Messungen gefundene außerordentlich hohe Beweglichkeit im Sinne der wellenmechanischen Metalltheorie zu deuten ist.
\end{abstract}

F ür das Verständnis der elektrischen Eigenschaften von Halbleitern erschien es zweckmäßig, die Größen, welche die elektrische Leitfähigkeit bestimmen, also Elektronenkonzentration und Elektronenbeweglichkeit, für ein halbleitendes Element aus Messungen des spezif. Widerstandes und des Hall-Effektes zu ermitteln und deren Beeinflussung durch Fremdmetallzusätze genau bekannter Größe zu studieren. Als Halbleiter wurde Germanium, als Zusatz Kupfer gewählt, also zwei nicht flüchtige Stoffe, so daß ein durch Synthese vorgegebenes Mengenverhältnis erhalten blieb. Die Untersuchungen sollten sich nur auf das Gebiet bis zu einem Kupfergehalt von 10 Gew.-\% erstrecken, in welchem keine Mischkristallbildung oder gegenseitige Löslichkeit zu erwarten ist ${ }^{1}$.

1 R. Schwarz u. G. Elstner, Z. anorg. allg. Chem. 217, 289 [1934].
Die Leitfähigkeit ist unter der Voraussetzung, daß nur eine Art freier Ladungsträger vorhanden ist und der Leiter als isotrop angesehen werden kann, durch

$$
\sigma=\frac{1}{\varrho}=n e b\left(\Omega^{-1} \mathrm{~cm}^{-1}\right)
$$

gegeben, wobei $n$ die Konzentration der Ladungsträger, $e$ die Ladung und $b$ die Beweglichkeit der geladenen Teilchen ist. Ist die am Präparat gemessene Querspannung $E_{H}$ (Volt), $d$ die vom Magnetfeld durchsetzte Schichtdicke in $\mathrm{cm}, J$ der Primärstrom in Ampère und $H$ die magnetische Feldstärke in Gauß, so ist die Hall-Konstante $R$ gegeben durch

$$
R=\frac{E_{H} d}{J H} \cdot 10^{8}\left(\mathrm{~cm}^{-3} / \mathrm{Amp} \mathrm{sec}\right) .
$$

Andererseits gilt nach der klassischen Statistik, 